\title{
La formación identitaria cultural local en el profesor de Geografía ${ }^{1}$
}

\author{
Master Rafael Jorge Hechavarría² \\ Dr. Jesús Piclín Minot ${ }^{3}$ \\ Dra. Raysa Hernández Batista ${ }^{4}$
}

\section{RESUMEN}

La formación de la identidad cultural local se dinamiza a partir de las propias necesidades socioeducativas de la sociedad que tienen expresión concreta en el Modelo de Formación del Profesional de Geografía. En este sentido, desde la presente investigación se ofrece una contribución teórica a la solución de este problema científico, a partir del análisis históricológico que profundiza en los antecedentes teóricos y metodológicos, así como la sistematización de los referentes para construir los fundamentos epistemológicos de este proceso, para lo cual se propone un metodología con el fin de favorecer la formación de la identidad cultural local desde el enfoque geohistórico en el contexto de la formación del profesor de Geografía.

Palabras clave: Formación, identidad cultural, enfoque geohistórico.

\section{Local cultural identity formation in the teacher of Geography}

\section{ABSTRACT}

The formation of local cultural identity is dynamized from the socio-educational needs of society that have concrete expression in the Training Model of the Geography Professional. In this sense from the present research offers a theoretical contribution to the solution of this scientific problem, from the historical-logical analysis, which delves into the theoretical and methodological background, as well as the systematization of the references to build the epistemological foundations of This process is proposed a methodology to promote the formation of local cultural identity from the geohistorical approach in the context of the formation of Geography teacher.

Keywords: Formation, cultural identity, geohistorical approach.

I Este artículo es resultado del proyecto de investigación "La evaluación de la calidad de la educación en las instituciones educativas del territorio guantanamero".

2 Master en Ciencias de la educación. Vicedecano de Investigación, Facultad de Ciencias de la Educación, Universidad de Guantánamo, Cuba. E-mail: Rafaelj@cug.co.cu

3 Doctor en Ciencias Pedagógicas. Director del Área de Información Científico-Técnica, Universidad de Guantánamo, Cuba. E-mail: jesusp@cug.co.cu

4 Doctor en Ciencias Pedagógicas. Profesor de formación doctoral, Universidad de Holguín, Cuba. E-mail raysa@femsu.uho.cu 


\section{Introducción}

La formación del profesor de Geografía debe propiciar la apropiación de aquellos contenidos geográficos que, por su potencialidad educativa, favorezcan la formación de la identidad cultural local, de modo que el estudiante en formación, en su actuación, logre no solo apreciar la belleza del paisaje natural sino también revelar los rasgos que le distinguen y que permiten se le ofrezca significatividad.

En el plano internacional son evidentes los presupuestos que asumen los intelectuales sobre el tema de estudio, tal es el caso de E. Díaz (s.p), A. Moreno (1998) y E. Rodríguez (2006), al considerar que es preciso introducir aires renovadores en la formación del profesor de Geografía, que le permita comprender las relaciones que se manifiestan en el espacio geográfico, al hacer una Geografía más explicativa, en la que los conocimientos no aparezcan fragmentados, aislados entre sí y sin conexión con la realidad.

Otros como E. Rodríguez (2010), O. De Cos (2010), P. Reques (20I0), X. Márquez (20I I), Y. Villegas (20I I) y M. Marrón (s.p), al abordar en la formación del profesorado la educación geográfica, son del criterio de incluir un determinado valor ético, que configure una actitud comprometida hacia el entorno natural y social en el que se desenvuelve la vida de la persona. Este valor podrá ser distinto en razón de los patrones culturales de cada pueblo o época, pero siempre deberá responder a una ética ambiental o geográfica, que presida las relaciones entre espacio, naturaleza y sociedad. A decir de esta relación, se considera prudente incorporar la cultura como componente esencial en el vínculo que establece el hombre con la naturaleza, en la satisfacción de sus necesidades, las cuales han estado más orientadas a las materiales que a las espirituales.

En el contexto cubano se destaca un grupo de autores como P. Recio (2009), C. Pérez (2008), R. Cuétara (2008), 2015), A. Hernández (20I2), E. Rodríguez (20I3), P. Álvarez (20I4, 20I5), J. Mateo (2015), A. Güidi (2017) y G. Guibert (2018), los cuales realizan, desde diversas perspectivas, un análisis epistemológico de la formación del profesional de Geografía. 
Al respecto destacan las investigaciones de C. Pérez (2008), R. Cuétara (2008), R. Hernández (2008), E. Rodríguez (2013) y J. Mateo (2015), al reconocer la función cultural que asume la Geografía, así como su contribución a la formación identitaria en los estudios locales. Sin embargo, se considera que las investigaciones precedentes dejan carencias teórico-prácticas que no permiten explicar la identidad cultural local en la formación del profesor de Geografía, al no considerar, desde el punto de vista pedagógicogeográfico, la integración del saber geográfico con el histórico local con fines identitarios, a partir de la preparación en el desarrollo de una educación geográfica que, como intencionalidad, posibilite la comprensión e interpretación del espacio geográfico local con un sentido de identidad.

En la localidad guantanamera es necesario relevar a los autores J. Piclín (2008) y G. Guibert (2018). El primero, al referirse a la geógrafa y pedagoga guantanamera Sarah Ysalgué, resalta el valor de su obra científico-educacional y su interés por la conformación de la identidad cultural local como finalidad y estímulo del desarrollo de la iniciativa y el establecimiento de normas metodológicas y direcciones organizativas que permitan la formación de la personalidad dentro del enfoque cultural del medio.

Sin embargo, la contribución de la geógrafa Sarah Ysalgué se queda al margen de los valores patrimoniales nacionales y universales: no siempre fueron suficientemente tratados en su obra los valores patrimoniales locales ni aparecen consideraciones didácticas en su obra sobre la formación de la identidad cultural local, en rescate de los valores más progresivos de la cultura autóctona y de la memoria histórico-local, a partir de los cuales es posible reproducir y explicar los complejos procesos de estructura espacio-temporal de la realidad geográfica local en que se inserta y desarrolla el estudiante de Geografía su actividad.

Por otra parte, G. Guibert (2018) realza las potencialidades formativas del contenido de la Geografía de Cuba en sus relaciones con Historia de Cuba, donde se identifica la formación identitaria como una de estas potencialidades para educar en el patriotismo. 
Con el objetivo de constatar el nivel de eficiencia con el que se desarrolla la formación de la identidad cultural local del profesor de Geografía, se realiza un diagnóstico fáctico que tiene en cuenta la revisión de documentos, la observación en clases, la aplicación de encuestas y entrevistas, la tutoría de trabajos de cursos y diplomas, cursos impartidos en eventos y otras vivencias investigativas que, como fruto de la experiencia profesional del autor de esta tesis, permiten identificar las siguientes insuficiencias, manifestadas en:

- La preparación que recibe el profesor de Geografía durante su formación no le permite apropiarse en su totalidad de la identidad cultural local desde la integración de saberes geográficos e históricos locales.

- Las concepciones establecidas acerca de la educación geográfica no siempre posibilitan al profesor en ejercicio identificar las potencialidades educativas del contenido geográfico para la comprensión e interpretación de los objetos geográficos como objetos de identidad.

- En los documentos normativos que rigen la formación del profesional de Geografía, son limitadas las indicaciones y orientaciones metodológicas que se ofrecen en el tratamiento de la identidad cultural local en el proceso de enseñanza aprendizaje de la Geografía.

En la sistematización teórica realizada por este investigador se evidencia que la integración del saber geográfico con el histórico local tiene una decisiva importancia para la organización y estructuración de las tareas en función de la identidad cultural local, razón por la cual será elaborado y utilizado en la propuesta que se ofrece a partir de los aportes del enfoque geohistórico en la integración del saber geográfico con el histórico, la convergencia con los aspectos centrales y de mayor relevancia en la teoría sobre identidad y la cultura, la articulación de la educación geográfica y la educación identitaria, así como la relación educación-culturacontexto-identidad.

Este análisis promueve la necesidad de ofrecer una metodología que favorezca la formación de la identidad cultural local. Por lo que, 
a criterio de este autor, los resultados investigativos anteriores no han sido determinantes en su totalidad para que el profesor que se forma en la especialidad Educación Geografía logre apropiarse del objeto geográfico local como un objeto geográfico de identidad local.

\section{Desarrollo}

\section{La identidad cultural local y su expresión en el Modelo del Profesional de la especialidad Educación Geografía}

Para Guibert (2018), la Geografía ocupa un lugar privilegiado entre las ciencias; no hay otra donde coexistan a la vez las ramas naturales y las sociales, de ahí su valor formativo-cultural, por la contribución al análisis y comprensión de los fenómenos complejos sobre el planeta Tierra y los que se relacionan con el hombre.

Es así que la formación del profesor de Geografía se encuentra actualmente en un cuadro complejo, determinado esencialmente por una ciencia geográfica que continuamente se reinterpreta -con enfoques derivados de la división epistémica de su objeto de estudioen lo natural y lo social, analizados desde la geografía física y la geografía humana, respectivamente (Álvarez, 2015).

Se concuerda con la profesora Guibert (2018) al justificar la diversidad de enfoques que han tenido expresión concreta en la formación del profesor de Geografía, "como consecuencia de la versatilidad de su fundamentación epistémica como ciencia de síntesis en correspondencia con los fundamentos del currículo".

Por tanto, el análisis de diversas fuentes de información corrobora la necesidad de elevar la calidad en la formación del profesional de Geografía, de modo que le permita, desde su actuación, estar preparado para enfrentar los problemas que en la práctica educativa se le presentan.

En este sentido, el actual Modelo del Profesional de la Especialidad: Educación Geografía responde al Plan de Estudio E y es el fruto de todo un proceso de retroalimentación de los anteriores planes de 
estudio y modelos de formación, por lo que una breve apreciación del actual modelo permite argumentar que:

- Desde los fundamentos teóricos que sustentan la formación del profesional se destacan ideas rectoras: la unidad entre la educación y la instrucción, la vinculación del estudio con el trabajo como principio pedagógico, la unidad dialéctica de la teoría con la práctica en el proceso de formación profesional, la relación naturaleza-sociedad y la relación entre la educación geográfica y la educación para la educación ambiental, el desarrollo sostenible y la cooperación internacional.

- Las indicaciones metodológicas y de organización de la carrera del referido Plan E precisa que: "La Geografía también tiene amplias posibilidades de contribución al estudio de la Historia de Cuba, pues en el país abundan los sitios geográficos de interés histórico". Así se reconoce a la Geografía de Cuba y la Historia de Cuba como las disciplinas académicas de mayores potencialidades para educar en los valores desde los estudios locales.

De este modo, una mirada desde el contexto de la investigación posibilita tener en cuenta dos aspectos importantes:

- Existe un acercamiento a la identidad cultural local desde la expresión de la identidad profesional como cualidad expresada en el modo de actuar de este profesional, a partir de demostrar el amor a la profesión y al ser humano, que se expresa en sentimientos de orgullo y de pertenencia a la profesión de enseñar Geografía.

- Además, en la función docente metodológica se asume como tarea la dirección del proceso de enseñanza-aprendizaje de la Geografía con enfoque interdisciplinario, estético, ético y axiológico, de manera que favorezca el cultivo de la sensibilidad, la espiritualidad y la formación de sentimientos, cualidades, valores, convicciones e ideales de nuestra sociedad socialista, lo que posibilita el tratamiento a la identidad cultural local.

A modo de resumen, en los últimos años en Cuba la formación del profesor de Geografía en el contexto de esta investigación se ha 
caracterizado por la falta de enfoques que orienten desde el Modelo del Profesional de Geografía la formación de la identidad cultural local y el poco aprovechamiento de las potencialidades educativas que ofrece el contenido geográfico de enseñanza en función de la identidad cultural local a partir de la integración de saberes.

\section{Referentes epistemológicos que sirven de base a la formación de la identidad cultural local en la formación inicial del profesor de Geografía}

Con el desarrollo y transformaciones que experimenta la sociedad contemporánea, la formación del profesional se convierte en un elemento imprescindible en la búsqueda de un profesor cada día más competente, en condiciones de dar respuesta a las demandas de la sociedad. En este sentido, la categoría "formación" ha sido abordada por autores como Bozhovich (1975), B. Honore (I980), C. Álvarez (I994), J. Chávez (I996), E. Baxter (2002), J. López (2005), P. Horrutinier (2007), R. Bermúdez, (20I4) y L. Pérez, (20I4), A. Alejandro (20I2), F. Addine (2013), G. Guibert (2018), entre otros.

En el marco conceptual de la pedagogía cubana, autores como C. Zayas (1994) y P. Horrutinier (2007) ofrecen sus consideraciones: "la formación es el proceso y el resultado cuya función es preparar al hombre en todos los aspectos de su personalidad”. Por otra parte, "se emplea para caracterizar el proceso sustantivo desarrollado en las universidades con el objetivo de preparar integralmente al estudiante en una determinada carrera universitaria y abarca, tanto los estudios de pregrado (o de grado, como se le denomina en algunos países) como los de posgrado".

Formar profesores es educarlos en su identidad hacia la profesión, es aportar la cultura en el enseñar, en la planificación de una adecuada concepción metodológica, en estrecho vínculo entre comunicación educativa y actividad pedagógica, que devienen como lógica a seguir y orientan la actividad y vías investigativas hacia sus diferentes partes como un todo coherente.

Por lo que se entiende que la formación del profesor de Geografía, en el contexto pedagógico, es un proceso sistemático y continuo, que 
contribuye a la preparación integral del profesor en formación, así como de su identidad hacia la profesión a partir de la adquisición de saberes, habilidades y valores que le permitan asumir una actuación profesional pedagógica, en la búsqueda de respuestas a los problemas que se le plantean en su desempeño y a las exigencias socioeducativas que emanan de la sociedad.

De este modo, la preparación pedagógica del profesor en formación es expresión de la interpenetración de lo ético, lo filosófico, lo histórico, lo estético, lo económico, lo artístico, lo político, lo ecológico, lo científico, lo tecnológico, lo pedagógico, entre otros aspectos que permitan la formación de una cultura integral. Sin embargo, las sociedad demanda un profesor que, a partir de los aspectos antes tratados, sea un contribuyente y defensor de la identidad cultural local.

Se reconocen los aportes que en el orden epistemológico se ofrecen de la identidad cultural local. Al respecto, Córdova (2008) considera que

La identidad cultural es un complejo fenómeno sociopsicológico con característica histórico-cultural, que se expresa desde las más simples manifestaciones de la vida cotidiana: prácticas culinarias, ajuares domésticos, vestuarios; se refleja en las variantes lingüísticas, idiosincrasia, relaciones familiares y sociales, etc.; se afirma en las costumbres, tradiciones, leyendas y folklore; se define a través de las producciones artísticas, literarias, históricas, pedagógicas, políticas y científicas en general; para alcanzar niveles superiores en la formación de la nacionalidad y llega a su madurez con la consolidación de una nación soberana.

El mexicano M. Rojas (2008) es del criterio que "es una categoría omnicomprensiva y compleja, que como identidad en la diferencia contiene, en correlación, la mismidad y la alteridad, el yo y el otro; (...), la cual caracteriza la manera común de vivir en el tiempo y el espacio del ser humano; (...), respondiendo a las preguntas qué he sido, qué soy y qué papel habré de desempeñar en el presente y futuro".

En el caso de C. Baeza (1996) y M. García (I996), es la producción de respuestas y valores que, como heredero y trasmisor, actor y 
autor de su cultura, el individuo realiza en un contexto histórico dado, como consecuencia del principio sociopsicológico de diferenciación-identificación en relación con otro(s) grupo(s) o sujeto(s) culturalmente definido(s). Estos autores analizan el proceso identitario y sus relaciones con la cultura, en la que el sujeto de la cultura, en la comunicación con otros significativos, a través de la actividad identitaria, es capaz de decodificar, codificar y transformar los mensajes culturales que recibe y responder a ellos, lo que, a partir de su diferenciación respecto del otro significativo e identificación con el mismo, deviene en su actuación sujeto de identidad.

Además tienen en cuenta la continuidad histórica del proceso identitario, al demostrar la vigencia en el tiempo de los objetos de identidad de acuerdo a su funcionalidad cultural. Por otro lado, estos objetos de identidad, cuando pierden su intención comunicativa, pasan a formar parte de la memoria histórico-cultural y son potencialmente utilizables por el sujeto de la cultura en ulteriores proceso identitarios.

El objeto de identidad, desde esta perspectiva, es comprendido como las producciones materiales y espirituales del sujeto de identidad, es decir, como el subconjunto de los objetos de la cultura que cumplen una función comunicativa actual entre un sujeto de la cultura dado y otro significativo (que en este caso se comporta como deviene, sujeto de identidad), bien por haber sido creados o refuncionalizados con ese propósito específico, bien por haber sido signados con una intención comunicativa determinada.

Todo lo anterior conduce a identificar tres aspectos importantes en la identidad cultural local: el modo de relacionarse los sujetos de la cultura y el sujeto de la cultura con el contexto local, la aprehensión que se ha hecho de la cultura local y la expresión de los conocimientos y valores a partir de la actuación y comunicación.

Esta visión integral de la identidad cultural local contribuye a ver patrones culturales en la Geografía que permiten revelar su función cultural y de identidad local. El geógrafo R. Cuétara (I998) advierte: "Constituye una verdad conocida en nuestro quehacer profesional, que la literatura geográfica ha dedicado más espacio a 
teorizar sobre el contenido, que a las vías por las cuales el escolar se apropia del conocimiento en la realidad circundante, habida cuenta de que ese entorno constituye, en sí mismo, la geografía del lugar que habitamos".

En el caso de Y. Labrada (2016), A. Parada (2016) y L. García (20I6) consideran que: "el tratamiento a los contenidos de la Geografía local no siempre forma parte de la preparación sistemática; falta incorporación de elementos locales contextualizados a las asignaturas y orientaciones metodológicas. Las actividades que se desarrollan en función de la formación permanente requieren de variabilidad".

Se precisa destacar que no solo se trata de contextualizar elementos geográficos locales, sino también, desde la contextualización, garantizar un proceso más interpretativo cultural del espacio geográfico local, que permita identificar los objetos geográficos que, por sus características, logren formar parte de la cultura y la identidad que se ha gestado en la localidad. Esta manera de percibir el espacio geográfico permite comprender que, como categoría fundamental de la Geografía, ha tenido diversas acepciones a partir de los enfoques que la han orientado como ciencia, entre otros autores se tiene a M. Santos (200I), T. Rojas (2005), R. Correa (2012) y M. Mateo (2015), que consideran al espacio como producto socialhumanizado, que se forma a partir de la relación de dos elementos fundamentales: lo natural y lo social.

Se concuerda con Mateo (2015), al plantear que el espacio geográfico es el soporte de unos sistemas de relaciones, donde unas se determinan a partir de los elementos del medio físico y otras procedentes de las sociedades humanas que ordenan el espacio en función de la densidad del poblamiento, de la organización social y económica, del nivel de las técnicas, de los significados que tiene para la sociedad; en una palabra, todo el tejido histórico que constituye una civilización.

La comprensión del espacio geográfico permite conocer, comparar y contrastar los objetos, procesos, fenómenos y hechos que ocurren en cada lugar en el contexto pedagógico, el estudiante, al explorar, recopilar y analizar la información espacial para diferenciar los lugares 
en diferentes escalas (local, regional, nacional, y global), configura su propia cultura, que en el escenario educativo de la Geografía es la cultura geográfica, lo que le lleva a conclusiones previas y tomar decisiones. Así, la Geografía es parte de la cultura e identidad del sujeto, "cultura que está articulada a espacios concretos de realización de los individuos, de los grupos sociales y de las sociedades". Unido a que

Las categorías geográficas están cargadas de símbolos y afectividad, son soporte de la identidad; o sea, de la cultura interiorizada por los individuos aislados y en colectividad.

Por otra parte, quizás el aspecto más conocido y a la vez el más diversamente interpretado del pensamiento geográfico ha sido el de su construcción como fenómeno cultural. En el proceso de transformación de la superficie terrestre, de su construcción y reconstrucción por la acción colectiva de los seres humanos, son creados y recreados modos de relación de la sociedad con el medio natural y social, y con los otros seres humanos, y se va produciendo cultura. Esto significa que la ocupación, la apropiación y transformación del espacio geográfico es un proceso cultural $(\ldots)$.

El espacio geográfico atesora objetos naturales que, por sus características, comprensión y apropiación por el sujeto de la cultura, posibilita o no la formación de la identidad cultural local. De ahí que el análisis del término "apropiación" sea de interés pedagógico. Al respecto se destacan autores como J. Zilberstein (2002), J. Chávez (2005), Fariñas (2005), Núñez (2006), J. Espinoza (2008), H. Fuentes (20II), J. Andrade (2015) y, desde sus posiciones, lo asocian con la formación del profesional.

El ecuatoriano J. Andrade (20I5), al abordar la apropiación, es de la opinión que "es un proceso dinámico, en que los estudiantes, profesores y otros sujetos participantes en el proceso formativo, de manera intencional y sistemática, desarrollan y transforman su cultura en relación directa con el contexto de actuación profesional en el que se desenvuelven, lo que significa que a la vez que se apropian de la misma, la enriquecen a partir de las diversas situaciones profesionales a las que se van enfrentando en la práctica”. 
En la definición anterior se aprecia que en la apropiación de la cultura intervienen los conocimientos y afectos, los motivos e intereses, así como las particularidades propias de cada estudiante en función de su realidad y sus referentes culturales. En este proceso de internalización, el estudiante atribuye significado a su profesión, lo que cobra sentido pedagógico-profesional en su contexto de actuación.

Por otra parte, la introducción del enfoque geohistórico en el contexto pedagógico constituye un imperativo de las transformaciones que se deben emprender en la apropiación del objeto geográfico como parte de la formación de la identidad cultural local, y para ello es necesario transformar la enseñanza de la Geografía e incorporar innovaciones en el proceso que contribuyan a una educación geográfica en función de la identidad cultural local. Esto encuentra soporte teórico en los aportes de R. Tovar (I986), R. Santaella, (1986), E. Rodríguez (2006), J. Armando (s.p), A. María y C. Puerta (s.p) y G. Guibert (2018).

Desde el enfoque geohistórico, la integración de lo geográfico con lo histórico local conduce a pensar en dos ideas de la autora G. Guibert (2018): "en la formación inicial del profesor de Geografía se deben sentar las bases para un proceso de enseñanza y aprendizaje de establecimiento de relación de los contenidos geográficos con los históricos, lo cual contribuye a asegurar su rasgo formativo (...)”. Y la segunda "lleva consigo reflexionar sobre el espacio geográfico desde una visión interdisciplinaria, que articula los procesos diacrónicos y sincrónicos en una simultaneidad dialéctica que se torna dinámica a la vez que evoluciona y se transforma como una totalidad temporoespacial".

Se reconoce la importancia del enfoque geohistórico con fines formativos; sin embargo, en el contexto pedagógico cubano se corrobora que sus fundamentos no siempre encuentran espacio en la educación identitaria, de modo que permita al profesor en formación tener una visión más integral del espacio geográfico donde se establecen nexos geográficos e históricos de identidad cultural local.

La educación identitaria encuentra aportes en A. Laurencio (2002), E. Montiel (I998), J Fabelo (201 I), E. Medina (2012) y O. González 
(2015). Estos realzan el papel de la educación como mecanismo para formar en la identidad cultural. Fabelo (20I I) considera que la educación puede actuar como una especie de antídoto y de resguardo de identidades. Y ofrece dos sugerencias pedagógicas para educar en la identidad: una asociada a la búsqueda en nuestras historias y en los valores tradicionales de todo aquello rescatable; la otra se orienta a que la educación ha de estar dirigida a evitar la pobreza axiológica del ser humano, a elevarlo por encima de sus necesidades corporales, a sensibilizarlo ante los productos espirituales humanos.

Por ello, se concibe que la educación identitaria es un sistema de influencias educativas que ejerce la escuela a través del profesor como mediador cultural, con el objetivo de generar los conocimientos y los sentimientos pertinentes desde el enriquecimiento de la cultura local, al formar valores y actitudes para una conciencia que sea expresada a través de su actuación en compromisos y sentimientos de pertenencia por el contexto geográfico local.

\section{Metodología para favorecer la formación de la identidad cultural local en el proceso formativo del profesor de Geografía}

La metodología que se propone se caracteriza por:

- Flexibilidad: al articular y ser adaptada a los resultados del diagnóstico cultural identitario que se realiza a los profesores y a la problemática identificada respecto a su preparación para favorecer la apropiación de la identidad cultural local en los profesores en formación.

- Formativa, porque propicia la reafirmación de conocimientos y actitudes, promueve el intercambio y el debate profesional fundamentado en la integración de saberes, desarrollando la crítica y la autocrítica.

- Enriquecedora y transformadora: facilita elevar la calidad en el proceso de formación del profesor de Geografía. Lo que permite transformaciones en el modo de pensar y actuar en relación con el tema objeto de estudio. 
- Humanista-personológica: posibilita el desarrollo de las potencialidades de los participantes, con un enfoque integral, se tiene en cuenta el carácter activo del sujeto en su propio proceso de construcción del conocimiento geográfico en sus relaciones con los históricos locales en función de la identidad cultural local.

- Orientadora, al ofrecer una vía para la apropiación de la identidad cultural local a partir de la integración del saber geográfico con el histórico local, lo que se sustenta en el enfoque geohistórico.

Su carácter diferenciador de las metodologías ya existente radica en que se sustenta en el enfoque geohistórico y, desde esta visión, tiene en cuenta los subsistemas y componentes de la concepción teóricometodológica, aspectos que en su unidad dialéctica la estructuran como sistema.

El aparato conceptual en que se sustenta la metodología se orienta a las concepciones acerca del enfoque geohistórico, a los presupuestos básicos que en relación con la apropiación de la identidad cultural local se establecen, así como a los criterios respecto de la metodología en su instrumentación práctica; además de las categorías que en el propio proceso investigativo se revelan, las cuales han sido tratadas en los capítulos anteriores.

\section{Etapas y momentos para su implementación}

Por tanto, se hace necesario establecer las etapas con sus respectivos procedimientos, con vista a la preparación del profesor de Geografía, para favorecer la formación de la identidad cultural local.

\section{Etapa 1. Creación de las condiciones previas.}

Objetivo: constatación de la existencia del problema científico y la factibilidad de favorecer su solución desde la preparación del profesor, en función de la apropiación de la identidad cultural local en la formación del profesor de Geografía.

Primer momento: diagnóstico de las necesidades y potencialidades de los sujetos que participan en el proceso de apropiación de la 
identidad cultural local en los profesores de Geografía en formación, que constará de dos espacios:

I. Un espacio de reflexión sobre la práctica habitual, con el objetivo de identificar experiencias y enfoques seguidos en función de la identidad cultural local.

2. Un espacio que implica profundizar en el perfil dinamizador del docente de Geografía por el sistema de conocimientos que enseña, ligado al contexto geográfico local, de modo que le permita asumir con compromiso el problema en torno al cual gira y se desarrolla la metodología, así como al problema profesional al cual responde.

Luego se está en condiciones de dar a conocer las exigencias sociales y las razones que justifican la búsqueda de nuevas vías, en función de la formación de la identidad cultural local y la necesaria preparación del profesor de Geografía para lograrlo. Para ello se debe tener en cuenta la relación cultura-contexto-identidad, además de reflexionar sobre la educación identitaria y las potencialidades educativas de la Geografía desde el enfoque geohistórico.

\section{Etapa 2. Procederes del profesor de Geografía para favorecer la formación de la identidad cultural local desde el enfoque geohistórico.}

Se tiene como propósito definir los pasos metodológicos que permiten la transformación del objeto desde su estado real hasta el estado deseado. Es el momento de concretar las acciones metodológicas a desarrollar según las mejoras de las necesidades formativas identitarias determinadas en la etapa anterior.

Además de precisar los aspectos a tener en cuenta para favorecer la apropiación de la identidad cultural local en la actuación profesional del profesor de Geografía, se planifican tareas de aprendizaje que permitan dar salida a los procesos teóricos y metodológicos establecidos.

La etapa se estructura en tres momentos:

a. Preparación teórica del profesor de Geografía en relación con enfoques y concepciones que orientan la identidad cultural 
local en el contexto de la formación pedagógica del profesor de Geografía.

b. Reconocimiento de los objetos geográficos como objetos de identidad de significación geohistórica cultural identitaria.

c. Contextualización de los objetos geográficos de significación geohistórica identitaria en el contenido de enseñanza.

A continuación se describe cada una de ellos:

a) Preparación teórico-pedagógica del profesor de Geografía en relación con enfoques y concepciones que orientan la identidad cultural local en el contexto pedagógico.

En este espacio se procederá a la preparación teórica del profesor de Geografía mediante los talleres de reflexión vivencial, pues no se puede desestimar la experiencia pedagógica y cultural que a lo largo de la vida el profesor ha acumulado. A continuación se presentan propuestas de temas que pueden formar parte de la preparación teórica del profesor de Geografía en función de favorecer la formación de la identidad cultural local.

Constituye una necesidad que los profesores de Geografía comprendan y asuman las funciones que se describen a continuación, para orientar adecuadamente al profesor en formación al interactuar con los objetos geográficos de significación geohistórica cultural identitaria:

- El objeto geográfico como objeto de identidad en sí mismo: se refiere a la caracterización del elemento identitario, su clasificación en cuanto a contenido y forma, así como su ubicación en el contexto geográfico local.

- El disfrute con el objetos geográficos de significación geohistórica identitaria: capacidad que tiene los sujeto de la cultura que, en su interacción con el objeto geográfico como objeto de identidad, actúa como sujeto de identidad, al percibir y hacer suyo no solo la belleza de estos, sino también su singularidad, aspecto que condiciona su significatividad a partir de lo que representa para él.

- La asunción de los objetos geográficos de identidad local: se asocia con la apropiación, por parte de los sujetos de identidad, de la herencia geo-histórica-cultural que les ha sido trasmitida 
de generación en generación, y con la manera que hacen suyos los objetos geográficos de identidad representativos del contexto geográfico local.

En la comprensión de los objetos geográficos de identidad local intervienen componentes conceptuales y formales que posibilitan la interacción entre las esferas cognitivo-instrumental y afectivomotivacional. Los mismos se determinan a continuación:

- Aproximación al objeto geográfico como objeto de significación geohistórica identitaria para su reafirmación como cubano y guantanamero.

- Sensibilidad ante el objeto geográfico como un objeto de identidad, de modo que no se conformen como un componente de la cultura del contexto geográfico local que solo posibilita el enriquecimiento cultural y no la identidad local.

- Comunicación asertiva con otros sujetos de la cultura (estudiantes y otros profesores), para expresar con identidad los objetos geográficos de significación geohistórica con los cuales convive y enriquece sus vivencias diariamente.

- Lo interpretativo-valorativo en la cultura del contexto geográfico local amerita que el profesor conozca con profundidad los códigos culturales que tipifican el contexto local como unidad espacial, donde confluyen los saberes geográficos con los históricos locales que se entretejen junto a otros componentes de la cultura, lo que posibilita la interpretación y valoración con un nivel mayor de profundidad, con una posición partidista.

b) Reconocimiento del objeto geográfico local de significación geohistórica cultural identitaria como un objeto geográfico de identidad local.

Este momento precisará de los siguientes procederes:

- Identificar, a partir de la singularidad geográfica e histórica local del objeto geográfico, su significación geohistórica cultural identitaria.

- Evaluar el nivel de aceptación cultural que ha tenido el objeto geográfico para los sujetos de la cultura local. 
- Destacar los rasgos que, desde el punto de vista identitario, identifican al objeto geográfico en la cultura local.

- Incorporar como parte del discurso académico en la actuación profesional al objeto geográfico de significación geohistórica cultural identitaria como un objeto geográfico de identidad local.

c) Contextualización de los objetos geográficos de identidad local en el contenido de enseñanza.

De este modo, la contextualización de los objetos geográficos de significación geohistórica cultural identitaria precisa de los siguientes procedimientos:

- Dominio del contenido del programa analítico de la asignatura y del texto básico.

- Identificar en el contenido los conceptos ya sistematizados (precedentes) y los nuevos por asimilar.

- Evaluar las potencialidades educativas del contenido geográfico con las potencialidades geohistórico-culturales del contexto geográfico local.

- Determinación de las relaciones contextuales manifestadas entre el contenido objeto de estudio y el objeto geográfico representado en el contexto geográfico local.

- Determinar los aspectos que caracterizan al objeto geográfico en sus relaciones espacio-temporales con el hecho histórico local.

- Revelar la significación geohistórica identitaria del objeto geográfico devenido objeto de identidad

- Precisar las condiciones del contexto didáctico donde se va a impartir.

- Tener en cuenta las percepciones y puntos de vista, así como la experiencia cultural del sujeto que va a ser el centro de este proceso.

- Proceder a la contextualización a partir del diseño de tareas de aprendizaje desde un enfoque geohistórico en función de la identidad cultural local. 


\section{Etapa 3. Evaluación de la propuesta por los profesores de Geografía y las sugerencias para medir el impacto de la metodología.}

Su propósito es controlar y evaluar, de forma sistémica, el grado de satisfacción de los docentes en la solución de la problemática durante su proyección y las alternativas trazadas, lo que incluye corregir errores y estimular las mejores experiencias pedagógicas en relación con la educación geográfica en función de la identidad local.

Se trata de sistematizar, integrar y valorar la construcción reflexivovalorativa que realiza el profesor en función de la formación de la identidad cultural local, desde lo cognitivo geohistórico cultural identitario y lo transformativo identitario, aspecto que opera hacia la reafirmación de los elementos identitarios, en tanto potencia, y permite articular el contenido geográfico identitario con los objetos geográficos de identidad local, con un pensamiento sistémico y reflexivo de acciones para percibir, apreciar e interpretar desde lo cognitivo la problemática cultural identitaria.

\section{Conclusiones}

El análisis de los fundamentos de la formación de la identidad cultural local en el proceso formativo del profesor de Geografía evidencia que, desde el enfoque geohistórico, se ofrece un sustento que permite apreciar la belleza del paisaje natural y revelar su singularidad geográfica con un significativo componente identitario, en consecuencia con las exigencias sociales y el momento histórico.

De esta manera, la metodología se orienta hacia la preparación del profesor de Geografía, que dirige el proceso enseñanza-aprendizaje de la Geografía, de modo que favorezca la formación de la identidad cultural local en el proceso formativo, lo que se transfiere al logro de un modo de actuación profesional pedagógico identitario.

\section{Referencias bibliográficas}

Andrade, J. (2015). Dinámica formativa intercultural universitaria. Tesis de doctorado. Santiago de Cuba: Universidad de Oriente. 
Álvarez, P., Esther, C y Recio, P. (2015). La formación del profesor de Geografía en Cuba ante la pluralidad de enfoques epistemológicos de la ciencia geográfica: retos y perspectivas actuales. Revista Varona, 6l, I-I7.

Araujo, N. (1989). Apuntes sobre el significado del valor de la identidad cultural. Revista Unión UNEAC, II (8).

Baeza, C. (1996). Modelo teórico para la identidad cultural. La Habana: Edit. José Martí.

Rodríguez, A. (200I). Propuesta pedagógica para el trabajo con los valores dignidad e identidad nacional en el ISPH. Tesis en opción al título académico de Master. Holguín: UCP "José de La Luz y Caballero”.

Pérez, E. (1999). La educación en valores, papel de la escuela. Curso 24, Pedagogía. La Habana: Edit. José Martí.

Córdova, C. (2008). Axiología e Identidad. Centro de Estudios de Cultura e Identidad. Universidad "Oscar Lucero Moya” de Holguín. (Documento en soporte digital).

López R. (2004). Hacia una Didáctica de la Geografía local. La Habana: Editorial Pueblo y Educación.

García, A. (2002). Identidad e Investigación. Centro de Investigación y Desarrollo de la Cultura Cubana. La Habana: Edit. Juan Marinello.

Rodríguez, G. (2018). Concepción metodológica para el establecimiento de relaciones entre la Geografía de Cuba y la Historia de Cuba. Tesis en opción al grado científico Doctor en Ciencias Pedagógicas. UCP "Enrique José Varona”, La Habana.

Labrada, Y., Parada, A. y García L. (2016). La metodología. Necesidad en la formación permanente del profesor de Geografía. Revista Didasc@lia: D\&E. Publicación cooperada entre CEDUT- Las Tunas y CEdEGGranma, CUBA, $I I(3)$.

Laurencio, A. (2002). La bistoria local en la formación de la identidad nacional de los escolares de Secundaria Básica. Tesis en opción al título académico Doctor en Ciencias Pedagógicas. ISP “José de la Luz y Caballero”, Holguín.

Lobo, G. (20I I). La Historia local en el proceso interdisciplinario del Básico Curricular Historia Geografía de Venezuela en el Programa Nacional de Formación de Educadores. Tesis en opción al título académico Doctor en Ciencias Pedagógicas. IPLAC, La Habana.

Rodríguez, M. (2015). Teoría y Metodología de la Geografía. La Habana, Cuba: Editorial Universitaria:.

Morillo, N. y Sayago, A. (2010). Praxis didáctica desde enfoque geohistórico en el aprendizaje de la Geografía local. Acción Pedagógica, 19. 
Pérez Álvarez, C. (2002). Didáctica de la Geografía. Selección de temas. La Habana, Cuba: Editorial Pueblo y Educación.

Pupo, R. (2006). El bombre, la actividad bumana, la cultura y sus mediaciones fundamentales. Tesis en opción al grado académico de Doctor en Ciencias. Cuba: Universidad de La Habana.

Rodríguez, E. (20I3). La educación patrimonial en la formación inicial del profesional de la educación de la carrera Licenciatura en Educación. Biología-Geografía. Tesis en opción al grado científico de Doctor en Ciencias Pedagógicas. UCP “ Félix Varela”, Villa Clara.

Santaella, R. (2005). Pensamiento geohistórico de Ramón Adolfo Tovar López. Revista Geoenseñanza Universidad de Los Andes, San Cristóbal, Venezuela, 10 (I). Recuperado de http://www.redalyc.org/articulo. oa?id=36010I0I.

Tamayo, I. (2009). Potencialidades formativas del pensamiento de Fidel Castro Ruz para el desarrollo de la identidad cultural del maestro primario. Tesis en opción al grado científico de Doctor en Ciencias Pedagógicas. UCP “José De La Luz y Caballero", Holguín.

Tovar, R. (1995). Boletín Geobistórico N ${ }^{\circ}$ I. Caracas: Centro de Investigaciones Geodidácticas y de Aplicación Geodidáctica.

Zamora, R. (1994). Notas para un estudio de la identidad cultural cubana. La Habana, Cuba: Centro de Estudio de la Cultura Cubana.

Zilberstein J. y Portela R. (2002). Una concepción desarrolladora de la motivación y el aprendizaje de las ciencias. En II Congreso Internacional de Didáctica de las Ciencias. La Habana, Cuba: OREAL-UNESCO. 